\title{
Heart Risk Assessment for Mining Workers in Papua New Guinea. Summary Paper for Heart Assessment and Stratification
}

ISSN: 2578-0379

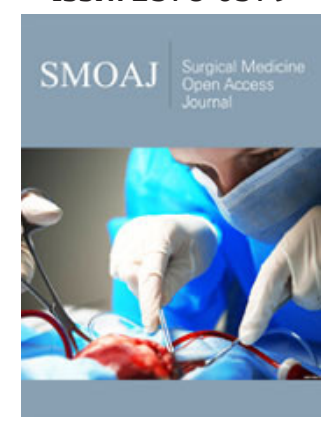

*Corresponding author: Arnold Waine, Division of Surgery, Papua New Guinea

Submission: 皆 February 24, 2020

Published: 制August 18, 2020

Volume 3 - Issue 4

How to cite this article: Arnold Waine. Heart Risk Assessment for Mining Workers in Papua New Guinea. Summary Paper for Heart Assessment and Stratification. Surg Med Open Acc J.3(4). SMOAJ.000568.2020. DOI: 10.31031/SMOAJ.2020.03.000568

Copyright@ Arnold Waine, This article is distributed under the terms of the Creative Commons Attribution 4.0 International License, which permits unrestricted use and redistribution provided that the original author and source are credited.

\author{
Arnold Waine* \\ Division of Surgery, Papua New Guinea
}

\section{Background/Introduction}

Papua New Guinea has about seven active mining and exploration activities for minerals like gold, copper, and other minor minerals. Each is managed by different company and together employs about ten thousand workers. A fifth of this would be foreign workers. Most of the Mine workers that are screened at the Employees Health and Wellness clinics tend to have similar compounding health risks. As a result, the common diseases that are prevalent are Hypertension, Diabetic Mellitus, High Cholesterol, general Obesity (BMI $>25 \mathrm{~m}^{2} / \mathrm{Kg}$ ) with minimal to zero level of physical exercise. Despite advice to religiously take the medications, there is noticeable lack of medication compliance, and a handful are usually under dosages.

While this provides a perfect niche for a cardiovascular disease of varying magnitude, there is grave concern for those who already developed severe hypertension and insidious progression to Ischemic Heart Diseases. Sudden myocardial infarction and/or heart attack, and cerebral vascular accident or stroke. These conditions do not usually provide preceding alarms, nor does it give you time to prepare, yet the end result is usually a permanent disability or worse, fatality. Therefore, the overall aim and objective of the Health and Wellness clinic established by each Mine sites is first to identify those people who are at high risk of developing heart diseases and initiate early treatment and lifestyle adjustment programs. Second aim of the clinic is to identify those who already are diagnosed with hypertensive or other vascular related medical conditions and require ongoing support and treatment. Thirdly for those with other medical conditions. The majority of the screening are for pre-work health and or routine follow up. However, for those that are in the second category, special health programs are initiated and continuous assessment about their condition is measured on a regular interval. Furthermore, a handful are referred to other major hospitals for specialist investigations and treatments.

\section{Employee Medical Assessment}

The Health and wellness clinic has a standard algorithm that consist of mostly objective parameters to measure all the compounding factors. Each categorical variable is given a nominal value to which a summative score is done, and health risk score is given. The category on the assessment includes age, gender, previous cardiac events, Body Mass Index BMI, Waist measurement, Blood cholesterol, lipids and glucose levels. Systolic Blood Pressure (SBP) and Diastolic Blood Pressure (DBP) and mean Arterial Pressure (MAP) are separately calculated. Physical exercise using Chester scoring system is also incorporated into the assessment form. Finally, the lifestyle habits like frequency of alcohol intake, smoke and Betel nut chewing (an area nut known as a stimulate and is used among local people) makes the final and important list for a major Cardiovascular event. A total score is given, and the Risk is calculated accordingly. While this medical assessment scoring system provides guide and assist in decision making on the management of the cases, it lacks specificity on its measurement on cardiovascular events. The reason is simply because of the dynamic flow of the blood, contents of the blood, the vascular wall integrity, and specific flow dynamics of 
coronary microvascular during diastole and overall strain on the myocardium. The Employee Health and Wellness clinics hereby propose to undertake further cardiovascular screening on high risk patients to yield increasing sensitivity and specificity on the subject and furthermore provides equivocal stratification. The combine algorithm extends to cover areas of bias and chances of hawthorn. It is the believe of this team that an objective measurable outcome is truly evaluated. Measurement that are reliable, reproducible and is easily performed without the need for expensive equipment. The following are the proposed methods specifically design to focus on the cardiovascular system of the mine workers in the country.

\section{Measurement}

\section{Endothelial glycocalyx}

The endothelium cell lining the vascular wall is covered with the protective glycocalyx. Composed of glycoproteins, proteoglycans, glycolipids and glycosaminoglycans of hyaluronic acid. The integrity of this substance plays a vital role in vascular permeability, inflammation, and elasticity [1,2]. Loss of glycocalyx has been shown to associate with hyperglycemia, hyperlipidemia, smoking, high blood pressure, and high sodium [3,4]. Damaged glycocalyx cumulatively causes impaired vascular elasticity, impaired coronary flow reserve, and myocardium deformation. Side stream Dark Field imaging is used to measure the perfused boundary region (PBR) of the sublingual arterial micro vessels. The thickness of the Glycocalyx is measured by this imaging using an instrument called the GlycoCheck. GlycoCheck is being formally adapted as a method to assess endothelial integrity by the European Society of Cardiology Working Group on Peripheral Circulation [4,5].

\section{Echocardiography}

Echocardiography (Using a Vivid 7 ultrasound system) is used to measure Left Ventricular myocardial deformation and the coronary flow reserve. Each of them is described according to the test done as below.

\section{Left Ventricular myocardial deformation}

Longitudinal systolic strain can be measured from standard 2-dimensional acquisition with the use of a dedicated software application and its Global Longitudinal strain can be measured from the apical chambers [6].

\section{Coronary flow reserve}

Coronary blood flow velocity profiles are usually done solely by color-guided pulse wave Doppler. And the coronary flow reserve is calculated as the ratio of peak diastolic velocity to resting peak diastolic velocity. The average measurement from 3 cardiac cycles is recorded $[7,8]$.

\section{Arterial stiffness}

Arterial Stiffness is measured by Pulse wave velocity (PWV) using tonometry by Compiler. Two noninvasive pressure sensors are normally used to record the carotids and femoral waveforms. The distance between the two is measured using a tape measure.
PWV is calculated as the distance between carotid to femoral site divided by transit time between waves [9-11]. The evidence from many studies have clearly demonstrated that, reduced endothelial glycocalyx thickness as assessed by the PBR, there is a corresponding increase of systolic blood pressure, and an increase Pulse Wave Velocity which denotes for arterial stiffness. Decrease glycocalyx is also associated with decreasing coronary flow reserve (CFR). In one study, multivariable regression analysis of the association of decreased glycocalyx thickness shows increased PBR, decreased CFR, and decrease PWV. It remains significant after readjustment of age, gender, BMI, Smoke, LV Mass, heart rate, hyperlipidemia [12].

\section{Conclusion}

This summary paper highlights the need for further assessment of Mine workers of Papua New Guinea with higher risk of cardiovascular events with specific screening modalities. This is to ascertain the myocardial strain and its overall function. To avoid catastrophic outcomes to the person, family and overall companies' establishment.

\section{Declaration}

Thank you to Dr Turhurus and team for the opportunity given to me to experience corporate health services and its dynamics during this time and my former visits. My travels and stay were all sponsored by the OK Tedi Mining Ltd through its Occupational Health and Safety division.

\section{References}

1. Noble MI, Drake HAJ, Vink H (2008) Hypothesis: Arterial glycocalyx dysfunction is the first step in the atherothrombotic process. QJM 101: 513-518.

2. Weinbaum S, Tarbell JM, Damiano ER (2007) The structure and function of the endothelial glycocalyx layer. Annu Rev Biomed Eng 9: 121-167.

3. Max N, Haeften TW, Gouverneur MC, Hans L Mooij, Miriam HPL, et al. (2006) Loss of endothelial glycocalyx during acute hyperglycemia coincides with endothelial dysfunction and coagulation activation in vivo. Diabetes 55(2): 480-486.

4. Lekakis J, Abraham P, Balbarini A, Andrew B, Chantal MB, et al. (2011) Methods for evaluating endothelial function: A position statement from the European society of cardiology working group on peripheral circulation. Eur J Cardiovasc Prev Rehabil 18(16): 775-789.

5. Mancia G, Fagard R, Narkiewicz K, Michel A, Michel B, et al. (2013) ESH/ ESC Guidelines for the management of arterial hypertension: ESH/ESC Guidelines for the management of arterial hypertension. Eur Heart J 39(33): 2159-2221.

6. Reisner SA, Lysyansky P, Agmon Y, Mutlak D, Lessick J, et al. (2004) Global longitudinal strain: A novel index of left ventricular systolic function. J Am Soc Echocardiogr 17(6): 630-633.

7. Ikonomidis I, Tzortzis S, Triantafyllidi H, John P, Costas P, et al. (2015) Association of Impaired left ventricular twisting-untwisting with vascular dysfunction, nuerohumoral activation and impaired exercise capacity in hypertensive heart disease. Eur J Heart Fail 17(12): 12401251.

8. Ikonomidis I, Lekakis J, Papadopoulos C, Helen T, Ioannis P, et al. (2008) Incremental value of pulse wave velocity in the determination of coronary microcirculatory dysfunction in never-treated patients with essential hypertension. Am J Hypertens 21(7): 806-813. 
9. Ikonomidis I, Ntai K, Kadoglou NP, John P, Ioannis P, et al. (2013) The evaluation of pulse wave velocity using arteriograph and complior apparatus across multiple cohorts of cardiovascular-related diseases. Int J Cardiol 168(5): 4890-4892.

10. Ikonomidis I, Makavos G, Lekakis J (2015) Arterial stiffness and coronary artery diseases. Curr Opin Cardiol 30(4): 422-431.

11. Tritakis V, Tzortzis S, Ikonomidis I, Kleanthi D, Georgios P, et al. (2016) Asociation of arterial stiffness with coronary flow reserve in revascularized coronary artery diseases patients. World J Cardiol 8(2): 231-239.

12. Ignatios I, Astrinos V, Geroge M, George Pavlidis, Dimitris Benas, et al. (2018) Association of impaired endothelial glycocalyx with arterial stiffness, coronary microcirculatory dysfunction, and abnormal myocardial deformation in untreated hypertensives. The Journal of clinical hypertension 20(4): 672-679. 